\title{
A Narrative Review of the Clinical Practicalities of Bamlanivimab and Etesevimab Antibody Therapies for SARS-CoV-2
}

\author{
Ramesh Nathan · Imad Shawa · Inmaculada De La Torre • \\ Jennifer M. Pustizzi · Natalie Haustrup (D) - Dipak R. Patel • \\ Gregory Huhn
}

Received: June 25, 2021 / Accepted: July 22, 2021 / Published online: August 10, 2021

(C) The Author(s) 2021

\begin{abstract}
The severity of coronavirus disease 2019 (COVID-19) ranges from mild to death, with high morbidity and mortality rates reported amongst a vulnerable subset of patients termed high risk. While vaccines remain the primary option for COVID-19 prevention, neutralizing monoclonal antibodies (mAbs), such as bamlanivimab and etesevimab, have been shown to benefit certain subpopulations after exposure to severe acute respiratory syndrome coronavirus 2 (SARS-CoV-2). Unlike vaccine-derived immunity that develops over time, administration of neutralizing mAbs is an immediate and passive immunotherapy, with the potential to reduce disease progression, emergency room visits, hospitalizations, and death. Bamlanivimab alone and together with etesevimab hold emergency use authorizations in several
\end{abstract}

R. Nathan

Los Robles Health System, Thousand Oaks, CA, USA

I. Shawa

Franciscan Health, 701E County Line Rd, Ste 101, Greenwood, IN 46143, USA

I. De La Torre · J. M. Pustizzi · N. Haustrup .

D. R. Patel

Eli Lilly and Company, Indianapolis, IN, USA

G. Huhn ( $\square)$

The Ruth M. Rothstein CORE Center, Cook County

Health and Hospital System, Chicago, IL, USA

e-mail: ghuhn@cookcountyhhs.org countries globally, with countries increasingly transitioning to the use of bamlanivimab and etesevimab together and other authorized mAbs on the basis of their evolving variant landscape, regulatory authorizations, and access to drugs. The current guidelines for the administration of bamlanivimab alone or together with etesevimab are informed by an iterative process of testing and development. Herein the rationale for these guidelines is provided by sharing the learnings that have been gathered throughout the development process of these mAbs. In addition, this review addresses the most common clinical questions received from health care professionals (HCPs) and patients regarding indicated population, dose, use with other medications and vaccines, duration of protection, and variants in clinical practice. As prevalence of SARS-CoV-2 variants can differ by country and state, prescribing HCPs should consider the prevalence of bamlanivimab and etesevimab resistant variants in their area, where data are available, regarding potential efficacy impact when considering treatment options.

Trial Registration: ClinicalTrials.gov identifier: $\quad$ NCT04427501; NCT04411628; NCT04497987; NCT04634409.

Keywords: Bamlanivimab; Clinical; COVID-19; Etesevimab; Health care practitioners; 
Monoclonal antibodies;
Treatment

\section{Key Summary Points}

Unlike vaccine-derived immunity that develops over time, administration of neutralizing monoclonal antibodies (mAbs) is an immediate and passive immunotherapy that reduces viral load and holds the potential to control disease progression and prevent emergency room visits, hospitalizations, and death.

This report describes the development of bamlanivimab and etesevimab, IgG1 human neutralizing $\mathrm{mAbs}$ that bind to distinct yet overlapping epitopes within the receptor binding domain of the SARSCoV-2 spike protein, and the role of bamlanivimab and etesevimab for the treatment of patients with mild-tomoderate COVID-19 illness within 10 days of symptom onset and at high risk for progressing to severe COVID-19 and/ or hospitalization.

Bamlanivimab alone and together with etesevimab hold emergency use authorizations in several countries globally, with countries increasingly transitioning to the use of bamlanivimab and etesevimab together and other authorized mAbs on the basis of their evolving variant landscape, regulatory authorizations, and access to drugs.

This report provides evidence-based practical guidance and rationales for administering bamlanivimab and etesevimab together and addresses the most frequent clinical questions received from health care professionals and patients around indicated population, dose, use with other medications and vaccines, duration of protection, and variants.
This report also discusses the practical learnings and the most recent real-world evidence detailing the adapted clinical care procedures and facilities that have been implemented to rapidly operationalize infusions of these mAbs.

\section{INTRODUCTION}

Prophylactic and therapeutic treatments are being developed to target the highly pathogenic severe acute respiratory syndrome coronavirus 2 (SARS-CoV-2) that is responsible for the ongoing coronavirus disease 2019 (COVID-19) global pandemic [1]. The spike protein of SARS-CoV-2 has become the focal point for many targeted treatment approaches, including neutralizing monoclonal antibodies (mAbs), which are recombinant proteins that can be used as a type of passive immunotherapy to minimize virulence [2]. While vaccines remain the primary option for COVID-19 prevention [3, 4], mAbs are an effective treatment for those already infected, as well as having the potential to prevent infection in those already exposed to SARSCoV-2, which can be of particular benefit to certain high-risk subpopulations [5-8]. Extensive reviews have been published on the role of mAbs in the COVID-19 treatment landscape with a focus on the mAbs that first received emergency use authorization (EUA) and their mechanism of action [5, 9]. Bamlanivimab became the first authorized therapeutic neutralizing $\mathrm{mAb}$ specifically developed to treat COVID-19 and was subsequently combined with a second $\mathrm{mAb}$, etesevimab $[6,10,11]$. Specifically, bamlanivimab and etesevimab are neutralizing immunoglobulin G1 (IgG1) mAbs that bind to distinct yet overlapping epitopes within the receptor binding domain (RBD) of the spike protein, thereby minimizing the potential for treatment-emergent resistant variants $[12,13]$. Other neutralizing $m A b s$ have also subsequently been granted EUAs, thus providing further evidence that this class of treatment 
is an effective targeted intervention for patients at risk $[14,15]$.

This review presents an overview of the treatment development of bamlanivimab and etesevimab together, provides the rationales for the clinical utility for patients, and examines the clinical practicalities of their administration in the rapidly changing disease landscape. The intention of this review is to address the most frequently received clinical questions from health care professionals (HCPs) and patients around the indicated population, dose, use with other medications and vaccines, duration of protection, and variants. This article is based on previously conducted studies and does not contain any new studies with human participants or animals performed by any of the authors.

This narrative review provides a summary of the main evidence-based practical guidance and rationales for administering bamlanivimab and etesevimab together and addresses the most frequently received clinical questions from HCPs and patients in six sections. The first section provides an overview of the treatment development process of bamlanivimab and etesevimab and is followed by a detailed review of the indicated population and the key clinical outcomes supporting the practical guidelines provided in the EUA factsheets. The subsequent section provides the evidence and rationales informing the infusion doses and timeframes. This section is followed by a brief review of the practical learnings gathered from the most recent real-world evidence of adapted clinical care procedures and facilities to rapidly operationalize infusions of these mAbs. The fifth section reviews the available information on concomitant medications and vaccines in relation to these mAbs. Finally, the evolving variant landscape is assessed and the implications for $\mathrm{mAb}$ treatments are discussed.

\section{OVERVIEW OF BAMLANIVIMAB AND ETESEVIMAB TREATMENT DEVELOPMENT}

The rapid development of bamlanivimab and etesevimab was contingent on the platform phase $2 / 3$ trial entitled BLAZE-1, an adaptive multiarm, multistage trial designed to allow evaluation of multiple interventions. An overview of the BLAZE-1 study design is provided in Fig. 1 . In the phase 2 portion of BLAZE-1, bamlanivimab was tested alone at three different doses (arms 2-4: 700, 2800, and $7000 \mathrm{mg}$, respectively) and in combination with etesevimab (arm 6: 2800/2800 mg). Learnings from phase 2 were subsequently applied in the phase 3 portion of BLAZE-1 where only patients at higher risk of developing severe COVID-19 were randomized to receive placebo or bamlanivimab and etesevimab together at two different dose combinations (arm 7: 2800/2800; arm 9: 700/1400 mg). Reduction in viral load, COVID-19-related hospitalizations and deaths, as well as symptomatology resolution were key outcomes and have been published elsewhere [13]. Table 1 summarizes the key efficacy results that further substantiated the suitability of bamlanivimab alone or together with etesevimab as treatment options for high-risk patients $[6,13,16]$.

The BLAZE-1 trial enrolled patients who had not been hospitalized, but had one or more mild or moderate COVID-19 symptoms [17]. Ambulatory patients (with mild-to-moderate COVID-19) that were at a higher risk of progressing to severe COVID-19 (based on the Centers for Disease Control and Prevention (CDC) guidance [18]) were infused within 3 days of providing their first positive SARSCoV-2 test sample. The median duration of symptoms was 4 days prior to infusion [13] and based on clinical practice and US Food and Drug Administration (FDA) guidance the factsheet indicates patients should be treated within 10 days of symptom onset [19]. Mild-to-moderate COVID-19 disease equates to a score of 1-4 on the World Health Organization (WHO) clinical progression scale that provides a measure of illness severity [20]. The majority of patients (77\%) enrolled to BLAZE-1 were classified as having mild COVID-19 defined by the FDA as symptoms of mild illness that could include cough, fever, sore throat, headache, malaise, muscle pain, diarrhea, nausea, vomiting, and no shortness of breath or dyspnea (per the protocol, patients with a saturation of 




Fig. 1 BLAZE-1 phase $2 / 3$ study design to evaluate the efficacy of bamlanivimab alone and together with etesevimab in ambulatory participants with mild to moderate

peripheral oxygen $\left(\mathrm{SpO}_{2}\right) \geq 93 \%$ or $\mathrm{PaO}_{2} /$ $\mathrm{FiO}_{2}>300$ were included), and no clinical signs indicative of moderate or severe, or critical severity [17].

As there is a risk of infusion reaction and hypersensitivity (including anaphylaxis) with any monoclonal agent, all trial participants were monitored closely during the intravenous (IV) infusion period and for at least $2 \mathrm{~h}$ following infusion completion [6]. On the basis of safety data accumulated since clinical studies began, monitoring following infusion completion has been reduced to approximately $1 \mathrm{~h}$. From phase 2 data, the most frequently reported adverse events reported in patients receiving bamlanivimab and etesevimab together (2800/ $2800 \mathrm{mg})$ or placebo were nausea $(3.6 \%$ and $3.8 \%$, respectively), and diarrhea $(0.9 \%$ and $4.5 \%$, respectively) [13]. The percentage of serious adverse events was $0 \%$ in the bamlanivimab monotherapy cohort compared with $0.9 \%$ in the bamlanivimab and etesevimab together cohort and $0.6 \%$ in the placebo cohort. Of the approximately 4000 subjects who have received bamlanivimab (either alone or with etesevimab) during BLAZE-1 and other clinical trials, approximately 800 subjects have received bamlanivimab and etesevimab together at the authorized doses $(700 / 1400 \mathrm{mg})$ during the phase 3 portion. Of the patients who have received bamlanivimab and etesevimab together at the authorized doses or higher, 1.1\% have had an infusion-related reaction and there
COVID-19 illness. Doses of bamlanivimab and etesevimab presented in brackets. $N$ number of patients in cohort

has only been a single case $(0.07 \%)$ of anaphylaxis [19].

Total evidence gathered from these clinical trials $[6,13]$ and supported by preclinical data [21] culminated in an EUA granted by the FDA in November 2020 for bamlanivimab alone [22] and subsequently for bamlanivimab and etesevimab together in February 2021 [19], which has also received a positive scientific opinion in the European Union (EU) from the committee for Medicinal Products for Human Use [23]. At the request of Eli Lilly and Company, the EUA for bamlanivimab alone has since been revoked by the FDA as of April 16, 2021, in response to the emergence of certain variants in the USA $[15,19,24]$. As of June 2021, three neutralizing monoclonal antibody (mAb) therapies (casirivimab and imdevimab together, sotrovimab alone, and bamlanivimab and etesevimab together) have EUAs in the USA [14, 15, 19]. Outside of the USA, bamlanivimab alone and bamlanivimab plus etesevimab hold EUAs, although these countries are transitioning to the use of bamlanivimab and etesevimab together and other authorized mAbs on the basis of their evolving variant landscape, regulatory authorizations, and access to drugs [15, 24]. Context and rationales for the guidance on the role of mAbs in the rapidly evolving variant landscape are provided herein to assist HCPs in making informed decisions. The key development milestones and clinical trials that lead to treatment authorizations and the increasing 
Table 1 Key clinical outcomes from the phase 2 and phase 3 portions of the BLAZE-1 trial of bamlanivimab and etesevimab together for patients with mild-to-moderate COVID-19

\begin{tabular}{|c|c|c|c|c|}
\hline & \multicolumn{4}{|c|}{ BLAZE-1 clinical trial } \\
\hline & \multicolumn{2}{|l|}{ Phase 2 portion } & \multicolumn{2}{|l|}{ Phase 3 portion $^{1}$} \\
\hline & $\begin{array}{l}\text { Bamlanivimab } \\
\text { and etesevimab } \\
(2800 / 2800 \mathrm{mg}) \\
\text { Entire cohort } \\
(N=112)\end{array}$ & $\begin{array}{l}\text { Placebo } \\
(N=156)\end{array}$ & $\begin{array}{l}\text { Bamlanivimab } \\
\text { and etesevimab } \\
(2800 / 2800 \mathrm{mg}) \\
\text { High-risk cohort } \\
(N=512)\end{array}$ & $\begin{array}{l}\text { Placebo } \\
(N=517)\end{array}$ \\
\hline \multicolumn{5}{|l|}{ Hospitalization, ED visits, and deaths } \\
\hline $\begin{array}{l}\text { Proportion of patients with COVID-19-related } \\
\text { hospitalization, ER visits, or deaths, \% }\end{array}$ & $\begin{array}{l}0.9 \\
p=0.075\end{array}$ & 5.8 & $\begin{array}{l}2.3 \\
p<0.001\end{array}$ & 7.0 \\
\hline Mean duration of hospitalization, days (SD) & 0 & $9.6(5.5)$ & $7.3(6.4)$ & $\begin{array}{l}11.2 \\
(10.1)\end{array}$ \\
\hline Deaths & 0 & 0 & 0 & 10 \\
\hline \multicolumn{5}{|l|}{ Viral load } \\
\hline $\begin{array}{l}\text { Change in log viral load from baseline to day } 7 \text {, } \\
\text { LSM (SE) }\end{array}$ & $\begin{array}{l}-3.78(0.175) \\
p<0.001\end{array}$ & $\begin{aligned} &- 2.66 \\
&(0.144)\end{aligned}$ & $\begin{array}{l}-3.66(0.090) \\
p<0.001\end{array}$ & $\begin{aligned} &- 2.46 \\
&(0.095)\end{aligned}$ \\
\hline \multicolumn{4}{|l|}{ Symptomology ${ }^{2}$} & \\
\hline $\begin{array}{l}\text { Change in symptomology viral load from baseline to } \\
\text { day 7, LSM (SE) }\end{array}$ & $\begin{array}{l}-4.19(0.287) \\
p<0.001\end{array}$ & $\begin{array}{r}-3.88 \\
(0.246)\end{array}$ & & \\
\hline Proportion of patients with symptomology & 45.9 & 40.8 & 34.4 & 26.5 \\
\hline Improvement at day $7, \%$ & & & & \\
\hline Proportion of patients with symptomology & 34.9 & 31.6 & 44.1 & 35.5 \\
\hline Resolution at day $7, \%$ & & & & \\
\hline Time to sustained symptomology resolution, days & 9 & 12 & 8 & 9 \\
\hline
\end{tabular}

$E R$ emergency room, $L S M$ least squares mean, $S E$ standard error, $S D$ standard deviation, $N$ number of patients in cohort, $n$ proportion of cohort, $p p$ value versus placebo

1 Endpoints differed in phase 2 and phase 3 portions of BLAZE-1 trial, therefore not all data available across phases. The median time to viral clearance is not available as less than $50 \%$ of each cohort of patients achieved viral clearance within the observation period ( 29 days). Phase 3 data for the patients who received bamlanivimab and etesevimab together (700/ $1400 \mathrm{mg})$ is not yet published

2 Symptom presence and severity of COVID-19 were assessed using a symptoms questionnaire and included symptoms of cough, shortness of breath, feeling feverish, fatigue, body aches and pain, sore throat, chills, headache, loss of appetite. Symptom severity was scored as $0,1,2$, or 3 corresponding to the symptom being absent, mild, moderate, or severe, respectively. Participants also rated changes in taste and smell with a yes/no response. Change in symptomology was evaluated on the basis of the total symptom questionnaire score (the sum of individual symptom scores). The definition of symptomatology resolution was an absence of all symptoms (phase 2), though it was amended for phase 3 data analysis to exclude a mild cough or fatigue 


\section{\begin{tabular}{|l|l|l|}
\hline \multicolumn{3}{|c|}{ Key } \\
\hline Phase 1 Trial & Phase 2 Trial & Phase 3 Trial \\
\hline
\end{tabular}}



Fig. 2 A timeline of the clinical development of bamlanivimab alone and together with etesevimab, including key milestones. EUA emergency use authorization, EMA
European Medicines Agency, CHMP EMA's human medicines committee, $H C P$ health care providers

health care providers should consider the benefit-to-risk ratio for an individual patient [19].

On the basis of clinical trials, bamlanivimab and etesevimab together have been identified as having an acceptable benefit-to-risk ratio for patients who are deemed to be at higher risk for progressing to severe COVID-19 and/or hospitalization $[13,19]$. "High risk" in the BLAZE trials was defined by the criteria derived from guidance originally provided by the CDC [18]. These criteria included an age of 65 years or older, a BMI of $35 \mathrm{~kg} / \mathrm{m}^{2}$ or more, or at least one relevant coexisting illness or concomitant medication. In the phase 2 portion of the BLAZE-1 trial, $67 \%$ of patients $(N=577)$ enrolled met at least one criteria for high risk; in the phase 3 portion of the BLAZE-1 trial, 100\% of patients $(N=1035)$ enrolled met the criteria for high risk [16]. In the phase 3 portion, the most common baseline high-risk comorbidities were age 65 years or older $(31.2 \%)$, BMI of $25 \mathrm{~kg} / \mathrm{m}^{2}$ or more $(86.4 \%)$, hypertension $(33.9 \%)$, and type 2 diabetes mellitus (18.8\%). Patients with chronic kidney disease, immunosuppressive treatments, immunosuppressive disease, chronic obstructive pulmonary disease, malignancies, and others were also included (each representing less than 5\%).

Consistently, there was a significant relative risk reduction (RRR) for COVID-related 


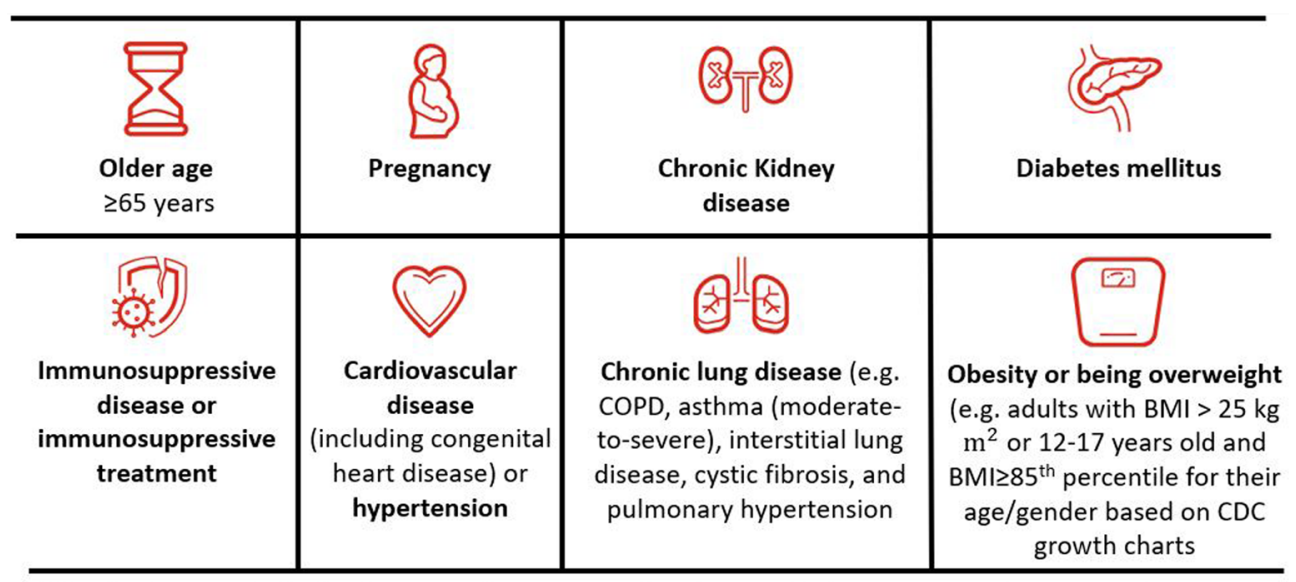

Other factors may include: sickle cell disease, neurodevelopmental disorders, including cerebral palsy or other conditions that confer medical complexity (e.g. genetic or metabolic syndromes) and medical-related technological dependence (e.g. tracheostomy, gastrostomy or positive pressure ventilation, not related to COVID-19. High risk could also be assigned if the patient met a combination of criteria, such as an age of 55 years or older as well as having cardiovascular disease, hypertension or a chronic respiratory disease.

Authorization of bamlanivimab with stessevimab under the EUA is not limited to the medical conditions or factors listed hers. Immunosuppressive diseases, as per the CDC guidelines, included having a solid organ



Fig. 3 Overview of high-risk criteria used to determine patient eligibility for treatment of mild-to-moderate COVID-19 with bamlanivimab and etesevimab together

hospitalization (at least $24 \mathrm{~h}$ of acute care) or death for patients who were treated with bamlanivimab and etesevimab across both phase 2 and phase 3 portions of BLAZE- 1 compared with patients who received placebo. In the phase 2 portion of BLAZE-1, the absolute risk reduction (ARR) and RRR for the entire cohort treated with bamlanivimab and etesevimab (2800/2800; $N=112$ ) were $5 \%$ and $100 \%$, respectively, compared with placebo and the number needed to treat (NNT) was 22. For the high-risk patient cohort treated with bamlanivimab and etesevimab $(2800 / 2800 ; N=38)$ the ARR and RRR were $9 \%$ and $100 \%$, respectively, compared with placebo and the NNT was 11. In the phase 3 portion of the BLAZE- 1 trial, the ARR and RRR for the entire high-risk patient cohort treated with bamlanivimab and etesevimab together $(2800 / 2800 \mathrm{mg} ; \mathrm{N}=518)$ were $5 \%$ and $70 \%$, respectively, compared with placebo and the NNT was 21. For the high-risk patients treated with bamlanivimab and etesevimab together $(700 / 1400 \mathrm{mg} ; N=511)$ the RRR was $87 \%$ compared with placebo. These data support the hypothesis that early intervention with bamlanivimab together with etesevimab greatly improves the clinical outcomes for high-risk ambulatory patients.

Pregnant or breastfeeding women were excluded from the clinical trials and therefore there are currently insufficient data to evaluate a drug-associated risk of major birth defects, miscarriage, or adverse maternal or fetal outcomes. Therefore, bamlanivimab and etesevimab should only be used during pregnancy if the potential benefit outweighs the potential risk for the mother and the fetus. From May 1, 2020 to February 26, 2021, 11 pregnancies were reported spontaneously in the bamlanivimab and etesevimab clinical trials, three of which were reported in patients treated with bamlanivimab and etesevimab together [33]. To date, there are no available data on the presence of bamlanivimab or etesevimab in human milk, the effects on the breastfed infant, or the effects on milk production.

Whilst there are limited data for the treatment of bamlanivimab and etesevimab together in pediatrics, the EUA recommendations were determined by the extrapolation of adult clinical trials based on weight models of outcomes. Although initial inclusion criteria for BLAZE-1 
specified that patients were 18 years or older, the age has since been reduced to 12 years or older and $1 \%$ of patients in the phase 3 portion $(2800 / 2800 \mathrm{mg})$ were $12-17$ years of age (inclusive) [34]. Children younger than 14 years old appear to be less commonly affected by COVID19 disease than adults, but the incidence increases with increasing age [35-39]. Despite reports of severe COVID-related illness, including fatality, in children, most children appear to be asymptomatic or report mild or moderate disease [40]. However, children with underlying medical conditions are at greater risk for severe COVID-19 disease compared with children without underlying conditions [38, 41].

\section{RATIONALES FOR INFUSION DOSE AND TIMEFRAME}

The authorized doses of bamlanivimab and etesevimab together $(700 / 1400 \mathrm{mg})$ were informed by pharmacokinetic (PK) and pharmacodynamic (PD) modeling, and antibody-viral dynamic modeling and simulations. The PD data showed a flat exposure-response relationship for efficacy within this dose range and the PK profile of bamlanivimab was also found to be linear and dose-proportional between 700 and $7000 \mathrm{mg}$ following a single IV administration [19]. In the phase 2 portion of BLAZE-1, pooled patients receiving any dose level of bamlanivimab $(700,2800,7000 \mathrm{mg})$ had a decreased frequency of ER visits and hospitalizations compared to patients that received placebo [6]. On the basis of these data, and to maximize the number of patients that could be treated with existing drug supply, the lowest dose of $700 \mathrm{mg}$ was selected for EUAs. To provide coverage of the different, but overlapping, epitopes on SARS-CoV-2 RBD sites for bamlanivimab and etesevimab together, the dose selection rationale for each single $\mathrm{mAb}$ administered together was the same as for a single $\mathrm{mAb}$ administered alone. On the basis of in vitro potency differences and PK/PD modeling, an approximately twofold higher etesevimab dose to bamlanivimab dose was judged to achieve maximum therapeutic response to reduce viral load, and sustained concentration above the respective $\mathrm{IC}_{90}$ of viral neutralization for at least 28 days in $90 \%$ of the patient population. Bamlanivimab with etesevimab $(700 / 1400 \mathrm{mg})$ also had similar antiviral activity compared with the higher dose combination (2800/ $2800 \mathrm{mg}$ ) in the phase 2 portion of BLAZE-1 [42]. On the basis of these data, the lowest tested dose of $700 \mathrm{mg}$ of bamlanivimab together with a twofold higher dose of $1400 \mathrm{mg}$ of etesevimab are now authorized [19].

One of the key learnings is that bamlanivimab alone or together with etesevimab should be administered as soon as possible after positive results of direct SARS-CoV-2 viral testing and within 10 days of symptom onset. The rationale for this timeframe is based primarily on the supportive efficacy and safety data collected for patients with mild-to-moderate COVID-19 symptoms during the BLAZE-1 trial, as opposed to patients who had already progressed to severe disease symptoms or had been hospitalized. Additionally, the ACTIV-3 trial undertaken by the National Institute of Allergy and Infectious Diseases (NIAID) has informed that neutralizing mAbs, on top of standard of care, might not be effective in the later stages of the disease when illness severity is driven primarily by the immune response, rather than active viral replication. Therefore, the use of bamlanivimab and etesevimab is limited to patients who are not hospitalized for COVID19 , nor require oxygen for COVID-19.

\section{REAL-WORLD EXPERIENCES}

As of April 28, 2021, the US Department of Health and Human Services reported that over 450,000 patients have received $\mathrm{mAbs}$ in the USA [43]. Despite the logistical challenge of administering IV infusions of mAbs to ambulatory, atrisk patients with COVID-19, there is growing independently published, real-world evidence that describes how different systems have operationalized the infusions based on multidisciplinary efforts and the repurposing of outpatient facilities [44-48]. Flexible referral systems and forms to test and identify suitable patients, as well as the coordinated collection of electronic real-time data have been 
examples of innovative approaches some hospitals, long-term care facilities, and outpatient or community-based centers have adopted in record time [44-48].

Real-world data based on case or control studies demonstrate that these neutralizing mAbs significantly reduce hospitalizations and deaths, without posing significant risks [46, 48-52]. Limited preliminary data has also indicated similar benefit may also apply for the most vulnerable populations, such as those with organ transplants [51].

On the basis of clinical trials and emerging real-world learnings, organizations have been able to adapt their systems to maximize the administration of neutralizing mAbs to patients. Key aspects facilitating greater flexibility for HCPs administering bamlanivimab and etesevimab include the aseptic preparation of the infusions based on simple dilutions and the administration of the drugs using different infusion bag materials (polyvinylchloride (PVC) or polyethylene (PE)-lined PVC), bag sizes (50, 100,150 , or $250 \mathrm{~mL}$ ), shortened infusion times (21-min infusion time when 50-mL infusion bag used), and pump or gravity infusion options [19]. There are also no hazardous risks associated with handling bamlanivimab and etesevimab and they can be stored for up to $7 \mathrm{~h}$ at room temperature or $24 \mathrm{~h}$ when refrigerated. In addition to these improvements, there is now a clearer understanding of monitoring requirements and how to handle potential adverse events (mainly mild to moderate infusion-related reactions). These factors have been essential to overcoming the challenges involved with treating patients with $\mathrm{mAb}$ treatments.

\section{CONCOMITANT MEDICATIONS AND VACCINES}

Inclusion criteria in the BLAZE-1 trial permitted certain medications to be used prior to the study including antivirals, corticosteroids, and other therapeutic agents [6]. During the study, certain concomitant medications were permitted if they were considered part of the local standard of care at the time. Up to $34 \%$ patients from the phase 2 portion of the study $(N=577)$ took one or more concomitant medications, including lopinavir, ritonavir, chloroquine, hydroxychloroquine, and corticoids. Acetaminophen and ibuprofen were the most commonly taken medications ( $20 \%$ and $8 \%$ ).

Convalescent COVID-19 plasma treatment prior to enrollment or during the study, participation in a previous SARS-CoV-2 vaccine study, or participation in a clinical study involving an investigational intervention within the last 30 days were not allowed per the exclusion criteria. Also, if a previous investigational intervention had a long half-life, then 5 half-lives or 30 days (whichever was longer) should have passed. Other exclusion criteria encompassed any serious concomitant systemic disease, condition, or disorder that, in the opinion of the investigator, would preclude participation in the study. Patients with moderate or severe hepatic impairment were also excluded, as evaluated with the criteria for hepatic dysfunction developed by the National Cancer Institute Organ Dysfunction Working Group [53]. Since there was no difference in the PK of bamlanivimab or etesevimab in patients with mild hepatic impairment compared with patients with normal hepatic function, no dosage adjustment is recommended in patients with mild hepatic impairment.

Concomitant use of medications with bamlanivimab and etesevimab is not contraindicated in the EUA as these mAbs are not renally excreted or metabolized by cytochrome P450 enzymes and therefore interactions are unlikely with concomitant medications. Data regarding the safety and efficacy of mAbs in patients with renal failure and patients receiving hemodialysis are limited, and most of the available information is based on case reports [54, 55]. As a result of the large size of the mAbs (146 kDa for bamlanivimab and $145 \mathrm{kDa}$ for etesevimab), they are unlikely to be affected by renal impairment or to be removed by hemodialysis $[22,55]$. Renal impairment and dialysis are not expected to impact the PK of bamlanivimab or etesevimab, and no dose adjustment is recommended in patients with renal impairment.

The BLAZE-1 trial excluded patients who had participated in a SARS-CoV-2 vaccine study; however, non-SARS-CoV-2 vaccinations were 
permitted prior to enrollment on the basis of standard of care or on a case-by-case basis following consultation with the sponsor [6]. Therefore, to date, there are no data available regarding the efficacy and safety of administration of a SARS-CoV-2 vaccine, or other vaccine, before or after receipt of bamlanivimab and etesevimab for the treatment of mild-to-moderate COVID-19. The mean apparent elimination half-life for bamlanivimab and etesevimab is 17.6 days and 25.1 days, respectively [19]. There is a theoretical risk that antibody administration may attenuate the endogenous immune response to SARS-CoV-2 and make patients more susceptible to reinfection [19].

For partially and fully vaccinated patients who subsequently develop COVID-19, prior receipt of a SARS-CoV-2 vaccine should not influence COVID-19 treatment decisions or the timing of such treatments $[56,57]$. Since there are no safety and efficacy data with COVID-19 vaccines in people who have received mAbs, the CDC recommends the deferral of vaccination for 90 days following antibody treatment as a precautionary measure until further data become available. The rationale for a 90-day deferral is not based on clinical trials but on general assumptions that take into account the estimated half-life of such therapies and the premise that reinfection is uncommon for 90 days after initial infection [58].

\section{IMPACT OF VARIANTS}

The SARS-CoV-2 virus is composed of four structural proteins, of which the spike glycoprotein is critical for viral attachment, fusion, and entry and therefore is a key target for antibodies and vaccines. The RBD unit of the spike protein mediates viral entry by binding to the angiotensin-converting enzyme 2 on the host cell, which is a cell receptor expressed by lung, gastrointestinal (GI) tract, and nasal mucosa cells. Naturally occurring variants in viruses are common with some mutations altering binding affinity and infectivity.

In order to predict and monitor for antiviral resistance, preclinical research was used to characterize the functional binding and to evaluate potential emergent treatment-resistant variants in vitro. Ongoing clinical research and global surveillance utilize genotyping and phenotyping to gain knowledge of resistance-associated mutations and the mechanisms of action of resistance. Viral genomes are continuously sequenced and shared via the Global Science Initiative on Sharing All Influenza Data (GISAID) (https://www.gisaid.org/), facilitating the real-time monitoring of genomic variants across the world. Since November 2020, the CDC carries out regular genomic surveillance across different US states to track emerging variants that may impact the efficacy of bamlanivimab, etesevimab, and other mAbs [59]. However, variant reporting is complex and available data can vary across databases because of changing naming or categorization conventions or whether the date recorded relates to when a sample was first collected or first submitted to a database [60-62]. Current estimates suggest the SARS-CoV-2 evolution rate is more comparable to influenza than measles, with the virus continuously evolving to escape immunity and therefore may require annually updated vaccines [63]. Variants of concern (VOC) and variants of interest (VOI) have emerged and been identified by the WHO since January 2020 [64]. These VOCs have similar mutations that might impact transmission, virulence, and treatment response, such as D614G and mutations at K417N/T, L452R, E484K, or N501Y. Particular mutations in the SARS-CoV-2 RBD domain can impact the binding of mAbs and thereby affect their efficacy.

The potential for SARS-CoV-2 to escape neutralization under selective pressure of bamlanivimab and/or etesevimab was studied in vitro using serial passaging and RBDdirected evolution studies. Passaging studies identified potential escape mutations for bamlanivimab (F490S and S494P) and etesevimab (D420N and N460K). RBD-directed evolution studies identified potential escape mutations for bamlanivimab (E484K/Q, F490S, Q493R, and S494P) and etesevimab (D420N and N460K/S) [65]. Significantly, no mutations were identified using either method under the selective pressure of bamlanivimab and etesevimab together. Clinical data also supported the assessment that 
combining neutralizing mAbs that target distinct epitopes within the RBD may minimize viral neutralization escape [13].

On the basis of the latest variant data, the EUA for bamlanivimab alone has been revoked by the FDA as of April 16, 2021 following a request by Eli Lilly and Company [22]. This revocation triggered an acceleration towards supply and use of bamlanivimab and etesevimab together in the USA, as well as outside of the USA (OUS). Bamlanivimab and etesevimab have both been found to have decreased binding to the Beta (formally South African origin, B1.351) and Gamma (formally Brazilian origin, P.1, B.1.1.28) variants $[19,64]$ whilst the everchanging variant landscape continues to be closely monitored as part of global surveillance efforts [66]. Currently, alternative authorized mAbs are available and are expected to retain activity against the Gamma and Beta variants [66]. Despite initial concerns regarding the Epsilon variant (formally California origin, B.1.429/B.1.427), recent pseudovirus neutralization data indicated that bamlanivimab together with etesevimab has only slightly reduced activity against the B.1.427/B.1.429 lineages or the L452R substitution found in this lineage [19]. Importantly, bamlanivimab and etesevimab together maintain potency against the Alpha variant (formally UK origin, B.1.1.7) as well as the rapidly spreading Delta variant (formally Indian origin, B.1.617.2) [60,67].

Neutralizing mAbs should be used on the basis of an assessment of the risk-to-benefit ratio, access, and variant situation in each specific case. Since there is the potential risk for treatment failure due to viral variants that are resistant to bamlanivimab, etesevimab, or other mAbs, it remains critical that HCPs only consider authorized mAbs that are expected to retain activity against the most common circulating viral variants in their area and to refer to the most updated factsheet in their countries.

\section{CONCLUSIONS}

As widespread vaccination campaigns gather pace and society moves towards ending the COVID-19 pandemic and eventual herd immunity, neutralizing monoclonal antibodies such as bamlanivimab and etesevimab continue to provide critical treatment options that can reduce the morbidity and mortality in high-risk patient groups. In response to the unprecedented global spread of COVID-19, bamlanivimab and etesevimab were developed rapidly and EUAs were granted within months of initiating trials. This review article shares the iterative learnings gathered during the trials of bamlanivimab and etesevimab and addresses the most frequent questions received from HCPs and patients regarding indicated population, dose, use with other medications and vaccines, duration of protection, and emerging variants. The prevalence of variants or mutations can differ from state to state and by country, and there is growing evidence to support that combinations of antibodies are less susceptible to viral resistance. It remains critical that HCPs only consider authorized mAbs that are expected to retain activity against most common circulating viral variants in their area and to refer to the most updated authorization factsheet in their countries and local jurisdictions. Finally, a summary is provided of the practical learnings provided by independent organizations who adapted procedures and facilities in an effort to rapidly operationalize infusions of these mAbs. Real-world evidence substantiating the efficacy and safety of these mAbs is also discussed. It is important to note, however, that this is a narrative style review rather than a systematic review and intends to provide HCPs with a comprehensive understanding of how to identify the role of mAbs for ambulatory, high-risk patients, and all of the clinical practicalities involved with administering bamlanivimab and etesevimab in the context of vaccines and variants. Open queries such as biomarkers of response and long-term benefit are still pending. Real-world studies, such as OPTIMISE-C19, will be critical in providing information on the long-term efficacy to prevent hospitalizations and mortality within the subgroups of high-risk patients as well as sustained symptomology resolution of monoclonal antibodies [68]. 


\section{ACKNOWLEDGEMENTS}

The authors would like to acknowledge the guidance provided by Christophe Sapin and Lisa Farmer Macpherson on statistical analyses. The authors also thank the investigators and support staff involved with the program, as well as the patients themselves. Bamlanivimab emerged from the collaboration between Eli Lilly and Company and AbCellera Biologics Inc. to create antibody therapies for the prevention and treatment of COVID-19. Eli Lilly and Company developed the antibody after it was discovered by AbCellera and scientists at the National Institute of Allergy and Infectious Diseases (NIAID) Vaccine Research Center. Etesevimab emerged from the collaboration among Eli Lilly and Company, Junshi Biosciences, and the Institute of Microbiology of the Chinese Academy of Sciences.

Funding. This work was supported by Eli Lilly and Company who is funding the journal Rapid Service Fee.

Medical Writing/Editorial assistance. Holly Green (Eli Lilly and Company) provided editorial assistance funded by Eli Lilly and Company.

Authorship. All named authors meet the International Committee of Medical Journal Editors (ICMJE) criteria for authorship for this article, take responsibility for the integrity of the work as a whole and have given their approval for this version to be published.

Author Contributions. Ramesh Nathan, Imad Shawa, Inmaculada De La Torre, Jennifer M. Pustizzi, Natalie Haustrup, Dipak R. Patel and Gregory Huhn interpreted the data and drafted the manuscript.

Disclosures. Inmaculada De La Torre, Dipak R. Patel and Jennifer M. Pustizzi are employees and stakeholders of Eli Lilly and Company. Natalie Haustrup is an employee of Eli Lilly and Company. Imad Shawa and Ramesh Nathan report grants from Eli Lilly and Company. Gregory Huhn reports grants and personal fees from Eli Lilly and Company and grants from Janssen, Viiv, Gilead and Proteus.

Compliance and Ethics Guidelines. This article is based on previously conducted studies and does not contain any new studies with human participants or animals performed by any of the authors.

Open Access. This article is licensed under a Creative Commons Attribution-NonCommercial 4.0 International License, which permits any non-commercial use, sharing, adaptation, distribution and reproduction in any medium or format, as long as you give appropriate credit to the original author(s) and the source, provide a link to the Creative Commons licence, and indicate if changes were made. The images or other third party material in this article are included in the article's Creative Commons licence, unless indicated otherwise in a credit line to the material. If material is not included in the article's Creative Commons licence and your intended use is not permitted by statutory regulation or exceeds the permitted use, you will need to obtain permission directly from the copyright holder. To view a copy of this licence, visit http://creativecommons.org/licenses/by$\mathrm{nc} / 4.0 /$.

\section{REFERENCES}

1. Hu B, Guo H, Zhou P, Shi Z-L. Characteristics of SARS-CoV-2 and COVID-19. Nat Rev Microbiol. 2021;19(3):141-54.

2. Renn A, Fu Y, Hu X, Hall MD, Simeonov A. Fruitful neutralizing antibody pipeline brings hope to defeat SARS-Cov-2. Trends Pharmacol Sci. 2020;41(11):815-29.

3. Krammer F. SARS-CoV-2 vaccines in development. Nature. 2020;586(7830):516-27.

4. Mello MM, Silverman RD, Omer SB. Ensuring uptake of vaccines against SARS-CoV-2. N Engl J Med. 2020;383(14):1296-9.

5. Marovich M, Mascola JR, Cohen MS. Monoclonal antibodies for prevention and treatment of COVID19. JAMA. 2020;324(2):131-2. 
6. Chen P, Nirula A, Heller B, et al. SARS-CoV-2 neutralizing antibody LY-CoV555 in outpatients with Covid-19. N Engl J Med. 2020;384(3):229-37.

7. Marston HD, Paules CI, Fauci AS. Monoclonal antibodies for emerging infectious diseases-borrowing from history. N Engl J Med. 2018;378(16): 1469-72.

8. Cohen MS, Nirula A, Mulligan MJ, et al. Effect of bamlanivimab vs placebo on incidence of COVID19 among residents and staff of skilled nursing and assisted living facilities: a randomized clinical trial. JAMA. 2021;326(1):46-55.

9. Taylor PC, Adams AC, Hufford MM, de la Torre I, Winthrop K, Gottlieb RL. Neutralizing monoclonal antibodies for treatment of COVID-19. Nat Rev Immunol. 2021;21(6):382-93.

10. Eli Lilly and Company. Lilly begins world's first study of a potential COVID-19 antibody treatment in humans [press release]. June 1, 2020. https:// investor.lilly.com/node/43276/pdf. Accessed 15 July 2021.

11. ClinicalTrials.gov. A study of LY3819253 (LYCoV555) in participants hospitalized for COVID-19. NCT04411628. 2020. https://clinicaltrials.gov/ct2/ history/NCT04411628. Accessed 15 July 2021.

12. Baum A, Fulton BO, Wloga E, et al. Antibody cocktail to SARS-CoV-2 spike protein prevents rapid mutational escape seen with individual antibodies. Science. 2020;369(6506):1014-8.

13. Gottlieb RL, Nirula A, Chen P, et al. Effect of bamlanivimab as monotherapy or in combination with etesevimab on viral load in patients with mild to moderate COVID-19: a randomized clinical trial. JAMA. 2021;325(7):632-44.

14. US Food and Drug Administration. Fact Sheet for health care providers: Emergency Use Authorization (EUA) of sotrovimab. 2021. https://www.fda. gov/media/149534/download. Accessed 15 July 2021.

15. US Food and Drug Administration. Fact sheet for health care providers: Emergency Use Authorization (EUA) of caririvimab and imdevimab. 2021. https://www.fda.gov/media/143892/ download2021. Accessed 15 July 2021.

16. Dougan $M$, Nirula $M$, Azizad $M$, et al. Bamlanivimab plus etesevimab in mild or moderate COVID-19. N Engl J Med. 2021. https://doi.org/10. 1056/NEJMoa2102685.

17. US Food and Drug Administration. COVID-19: developing drugs and biological products for treatment or prevention guidance for industry, Updated
February 22, 2021. 2020. https://www.fda.gov/ media/137926/download. Accessed 15 July 2021.

18. Centers for Disease Control and Prevention. People with certain medical conditions. Certain medical condititions and risk for severe COVID-19 illness. Updated November 2, 2020. https://www.cdc.gov/ coronavirus/2019-ncov/need-extra-precautions/ people-with-medical-conditions.html. Accessed 2 Mar 2021.

19. US Food and Drug Administration. Fact sheet for health care providers: Emergency Use Authorization (EUA) of bamlanivimab and etesevimab. 2021. https://www.fda.gov/media/145802/download. Accessed 9 July 2021.

20. Marshall JC, Murthy S, Diaz J, et al. A minimal common outcome measure set for COVID-19 clinical research. Lancet Infect Dis. 2020;20(8):e192-7.

21. Jones BE, Brown-Augsburger PL, Corbett KS, et al. The neutralizing antibody, LY-CoV555, protects against SARS-CoV-2 infection in nonhuman primates. Sci Transl Med. 2021;13(593):eabf1906.

22. US Food and Drug Administration. Fact sheet for health care providers: Emergency Use Authorization (EUA) of bamlanivimab. 2020. https://www. fda.gov/media/143603/download. Accessed 15 July 2021.

23. European Medical Agency. EMA issues advice on use of antibody combination (bamlanivimab/etesevimab). March 5, 2021. https://www.ema.europa. eu/en/news/ema-issues-advice-use-antibodycombination-bamlanivimab-etesevimabEMA. CHMP. Accessed 15 July 2021.

24. US Food and Drug Administration. Coronavirus (COVID-19) update: FDA authorizes additional monoclonal antibody for treatment of COVID-19. May 26, 2021. https://www.fda.gov/news-events/ press-announcements/coronavirus-covid-19update-fda-authorizes-additional-monoclonalantibody-treatment-covid-19. Accessed 15 July 2021.

25. Williamson EJ, Walker AJ, Bhaskaran K, et al. Factors associated with COVID-19-related death using OpenSAFELY. Nature. 2020;584(7821):430-6.

26. Ko JY, Danielson ML, Town M, et al. Risk factors for COVID-19-associated hospitalization: COVID-19associated hospitalization surveillance network and behavioral risk factor surveillance system. Clin Infect Dis. 2020;72(11):e695-703.

27. Petrilli CM, Jones SA, Yang J, et al. Factors associated with hospital admission and critical illness among 5279 people with coronavirus disease 2019 
in New York City: prospective cohort study. BMJ. 2020;369:m1966.

28. Jordan RE, Adab P, Cheng KK. Covid-19: risk factors for severe disease and death. BMJ. 2020;368:m1198.

29. Hariyanto TI, Putri C, Situmeang RFV, Kurniawan A. Dementia is a predictor for mortality outcome from coronavirus disease 2019 (COVID-19) infection. Eur Arch Psychiatry Clin Neurosci. 2021;271(2):393-5.

30. Kwenandar F, Japar KV, Damay V, et al. Coronavirus disease 2019 and cardiovascular system: a narrative review. IJC Heart Vasc. 2020;29: 100557.

31. Putri C, Hariyanto TI, Hananto JE, et al. Parkinson's disease may worsen outcomes from coronavirus disease 2019 (COVID-19) pneumonia in hospitalized patients: a systematic review, meta-analysis, and meta-regression. Parkinsonism Relat Disord. 2021;87:155-61.

32. Centers for Disease Control and Prevention. science brief: evidence used to update the list of underlying medical conditions that increase a person's risk of severe illness from COVID-19; Updated May 12, 2021. https://www.cdc.gov/coronavirus/2019ncov/science/science-briefs/underlying-evidencetable.html. Accessed 15 July 2021.

33. Eli Lilly and Company. Can bamlanivimab and etesevimab be used during pregnancy? https:// www.lillymedical.com/en-us/answers/canbamlanivimab-and-etesevimab-be-used-duringpregnancy-137197. Accessed 15 July 2021.

34. Eli Lilly and Company. What information is available regarding the use of bamlanivimab and etesevimab in pediatric patients? 2021. https://www. lillymedical.com/en-us/answers/what-informationis-available-regarding-the-use-of-bamlanivimaband-etesevimab-in-pediatric-patients-137194. Accessed 15 July 2021.

35. Dong Y, Mo X, Hu Y, et al. Epidemiology of COVID19 among children in China. Pediatrics. 2020;145(6): e20200702.

36. Lu X, Zhang L, Du H, et al. SARS-CoV-2 infection in children. N Engl J Med. 2020;382(17):1663-5.

37. Leidman E, Duca LM, Omura JD, Proia K, Stephens JW, Sauber-Schatz EK. COVID-19 trends among persons aged 0-24 years-United States, March 1-December 12, 2020. MMWR Morb Mortal Wkly Rep. 2021;70(3):88-94.

38. Götzinger F, Santiago-García B, Noguera-Julián A, et al. COVID-19 in children and adolescents in Europe: a multinational, multicentre cohort study. Lancet Child Adolesc Health. 2020;4(9):653-61.
39. Centers for Disease Control and Prevention. Clinical growth charts; updated June 16, 2017. https:// www.cdc.gov/growthcharts/clinical_charts.html. Accessed 10 Mar 2021.

40. Liguoro I, Pilotto C, Bonanni M, et al. SARS-COV-2 infection in children and newborns: a systematic review. Eur J Pediatr. 2020;179(7):1029-46.

41. Shekerdemian LS, Mahmood NR, Wolfe KK, et al. Characteristics and outcomes of children with coronavirus disease 2019 (COVID-19) infection admitted to US and Canadian pediatric intensive care units. JAMA Pediatr. 2020;174(9):868-73.

42. Eli Lilly and Company. Lilly's bamlanivimab and etesevimab together reduced hospitalizations and death in phase 3 trial for early COVID-19 [press release]. March 10, 2021. https://investor.lilly.com/ news-releases/news-release-details/lillysbamlanivimab-and-etesevimab-together-reduced. Accessed 15 July 2021.

43. Office of the Assistant Secretary for Preparedeness and Response. Update: Allocation and distribution of COVID-19 therapeutics. April 28, 2021. https:// www.healthy.arkansas.gov/images/uploads/pdf/ HHS_ASPR_Tx_Allocation_Distro_Stakeholder_ Updates_04.28.21_FINAL.pdf. Accessed 15 July 2021.

44. Bariola JR, McCreary EK, Khadem T, et al. Establishing a distribution network for COVID-19 monoclonal antibody therapy across a large health system during a global pandemic. Open Forum Infect Dis. 2021;8:ofab151.

45. Razonable RR, Aloia NCE, Anderson RJ, et al. A framework for outpatient infusion of antispike monoclonal antibodies to high-risk patients with mild-to-moderate coronavirus disease-19: The Mayo Clinic Model. Mayo Clin Proc. 2021;96(5): 1250-61.

46. Tulledge-Scheitel S, Bell SJ, Larsen JJ, et al. A mobile unit overcomes the challenges to monoclonal antibody infusion for COVID-19 in skilled care facilities. J Am Geriatr Soc. 2021;69(4):868-73.

47. Harris AK, Gibson AM, Spencer B, et al. Rapid implementation of a new ambulatory infusion location for patients with COVID-19 to receive monoclonal antibody therapy. Am J Health Syst Pharm. 2021;78(13):1166-8.

48. Kumar RN, Wu E-L, Stosor V, et al. Real-world experience of bamlanivimab for COVID-19: a casecontrol study. Clin Infect Dis. 2021; ciab305.

49. Bariola JR, McCreary EK, Wadas RJ, et al. Impact of bamlanivimab monoclonal antibody treatment on hospitalization and mortality among non- 
hospitalized adults with SARS-CoV-2 infection. Open Forum Infect Dis. 2021;8(7):ofab254.

50. Bariola JR, McCreary EK, Wadas RJ, et al. Impact of monoclonal antibody treatment on hospitalization and mortality among non-hospitalized adults with SARS-CoV-2 infection. medRxiv. 2021:03.25. 21254322.

51. Dhand A, Lobo SA, Wolfe K, Feola N, Nabors C. Bamlanivimab for treatment of COVID-19 in solid organ transplant recipients: early single-center experience. Clin Transplant. 2021;35(4): e14245.

52. Webb BJ, Buckel W, Vento T, et al. Real-world effectiveness and tolerability of monoclonal antibodies for ambulatory patients with early COVID19. medRxiv. 2021.03.15.21253646.

53. Elmeliegy M, Yang DZ, Salama E, Parivar K, Wang DD. Discordance between Child-Pugh and national cancer institute classifications for hepatic dysfunction: implications on dosing recommendations for oncology compounds. J Clin Pharmacol. 2021;61(1):105-15.

54. Jillella AP, Dainer PM, Kallab AM, Ustun C. Treatment of a patient with end-stage renal disease with rituximab: pharmacokinetic evaluation suggests rituximab is not eliminated by hemodialysis. Am J Hematol. 2002;71(3):219-22.

55. Meibohm B, Zhou H. Characterizing the impact of renal impairment on the clinical pharmacology of biologics. J Clin Pharmacol. 2012;52(1 Suppl): $54 s-62 s$.

56. Centers for Disease Control and Prevention. Understanding how vaccines work. 2018. https:// www.cdc.gov/vaccines/hcp/conversations/ understanding-vacc-work.html. Accessed 15 July 2021.

57. Centers for Disease Control and Prevention. Interim clinical considerations for use of mRNA COVID-19 vaccines currently authorized in the United States. February 10, 2021. https://www.cdc. gov/vaccines/covid-19/info-by-product/clinicalconsiderations.html. Accessed 15 July 2021.

58. Centers for Disease Control and Prevention. Interim clinical considerations for use of COVID-19 vaccines currently authorized in the United States. March 5, 2021 https://www.cdc.gov/vaccines/ covid-19/info-by-product/clinical-considerations. html. Accessed 15 July 2021.

59. Centers for Disease Control and Prevention. Genomic surveillance for SARS-CoV-2 variants. May 17,
2021. https://www.cdc.gov/coronavirus/2019ncov/variants/variant-surveillance.html. Accessed 15 July 2021.

60. Centers for Disease Control and Prevention. COVID-19 variant tracker. 2021. https://www.cdc. gov/coronavirus/2019-ncov/cases-updates/variantproportions.html Accessed 15 July 2021.

61. World Health Organization. Tracking SARS-CoV-2 variants. May 31, 2021. https://www.who.int/en/ activities/tracking-SARS-CoV-2-variants/. Accessed 15 July 2021.

62. O'Toole A, Hill V, Pybus OG, et al. PANGO: lineage assignment in an emerging pandemic as an epidemiological tool. https:/cov-lineages.org/ lineages.html. Accessed 4 June 2021.

63. Williams TC, Burgers WA. SARS-CoV-2 evolution and vaccines: cause for concern? Lancet Respir Med. $2021 ; 9(4): 333-5$.

64. World Health Organization. SARS-CoV-2 variants of concern and variants of interest. 2021. https:// www.who.int/en/activities/tracking-SARS-CoV-2variants/. Accessed 15 July 2021.

65. Kallewaard N, Heinz BA, Siegel RW, et al., editors. Variant selection, characterization and impact on antibody SARS-CoV-2 neutralization. In: Conference on retroviruses and opportunistic infections (CROI). 2021. https://www.croiconference.org/ abstract/variant-selection-characterization-andimpact-on-antibody-sars-cov-2-neutralization/. Accessed 15 July 2021.

66. US Department of Health and Human Services. Distribution of bamlanivimab/etesevimab paused to Arizona, California, Florida, Indiana, Oregon, and Washington in addition to Illinois and Massachusetts. May 26, 2021. https://www.phe.gov/ emergency/events/COVID19/investigation-MCM/ Bamlanivimab-etesevimab/Pages/default.aspx. Accessed 15 July 2021.

67. Planas D, Veyer D, Baidaliuk A, et al. Reduced sensitivity of infectious SARS-CoV-2 variant B.1.617.2 to monoclonal antibodies and sera from convalescent and vaccinated individuals. bioRxiv. 2021. https://doi.org/10.1101/2021.05.26.445838.

68. Huang DT, McCreary EK, Bariola JR, et al. The UPMC OPTIMISE-C19 (OPtimizing Treatment and Impact of Monoclonal antIbodieS through Evaluation for COVID-19) trial: a structured summary of a study protocol for an open-label, pragmatic, comparative effectiveness platform trial with responseadaptive randomization. Trials. 2021;22(1):1-3. 